\title{
Evaluation of low cost residual gas analyzers for ultrahigh vacuum applications
}

\author{
M. G. Rao a) and C. Dong \\ Thomas Jefferson National Accelerator Facility, Newport News, Virginia 23606
}

(Received 28 October 1996; accepted 6 January 1997)

In recent years several low cost computer controlled residual gas analyzers (RGAs) have been introduced into the market place. It would be very useful to know the performance characteristics of these RGAs in order to make an informed selection for UHV applications. The UHV applications include extreme sensitivity helium leak detection and monitoring of the residual gas spectra in UHV systems. In this article, the sensitivity and linearity data for nitrogen, hydrogen, and helium are presented in the pressure range $10^{-8}-10^{-1} \mathrm{~Pa}$. Further, the relationships between focus voltage and ion currents, relative sensitivity, and fragmentation factor are also included. A direct comparison method is used in obtaining this data. Spinning rotor and extractor gauges are the transfer standard gauges used in Jefferson Lab's vacuum calibration facility, with which all the reported measurements here were carried out. (C) 1997 American Vacuum Society.

[S0734-2101(97)53603-7]

\section{INTRODUCTION}

Residual gas analyzers (RGAs) have been continuously improved since their inception in the 1960s. The quadrupole type RGAs are widely used in accelerator, ${ }^{1}$ fusion facility, and various vacuum process based applications. The RGAs consist of an ion source, ion filtering, and ion detection subsystems. The operation of RGAs is complex and requires optimization of several electrical parameters that control the ion source and ion filtering systems for quantitative measurements. ${ }^{3-6}$ As a result, the users are likely to get different results based on the proper or improper selection of various operating parameters of the RGA. This user confusion in the proper use of RGAs has been the subject of discussion in recent literature. ${ }^{7-9}$

Researchers have spent great efforts to understand and find complex relationships between various electrical parameters of the ion source. As a result, it is clear that widely acceptable performance characteristics may not be achievable with various ion source structures for a given application. So users should have a complete acquaintance with the performance characteristics of their RGA. Users should also be aware bow the sensitivity and linearity of the RGA varies with different gas species and be comfortable in using an appropriate calibration method specific to their needs. The environmental sensitivity of the electron multiplier bas to be kept in mind for its long term successful use. The careful selection of various operating parameters is essential for acquiring useful data for a given application. Accurate and quantitative data can be obtained with RGAs with appropriate calibrations and the choice of suitable operating parameters.

In this article the results of our investigations on the linearity, sensitivities for different gas species, relative sensitivity, and its dependence on various parameters, instability of

a)Electronic nail: rao@jlab.org fragmentary patterns, and the necessary calibration techniques are presented for computer controlled RGAs.

\section{EXPERIMENTAL SETUP AND PROCEDURE}

The Jefferson Lab's vacuum gauge calibration facility shown in Fig. 1 is used for the measurements reported here.

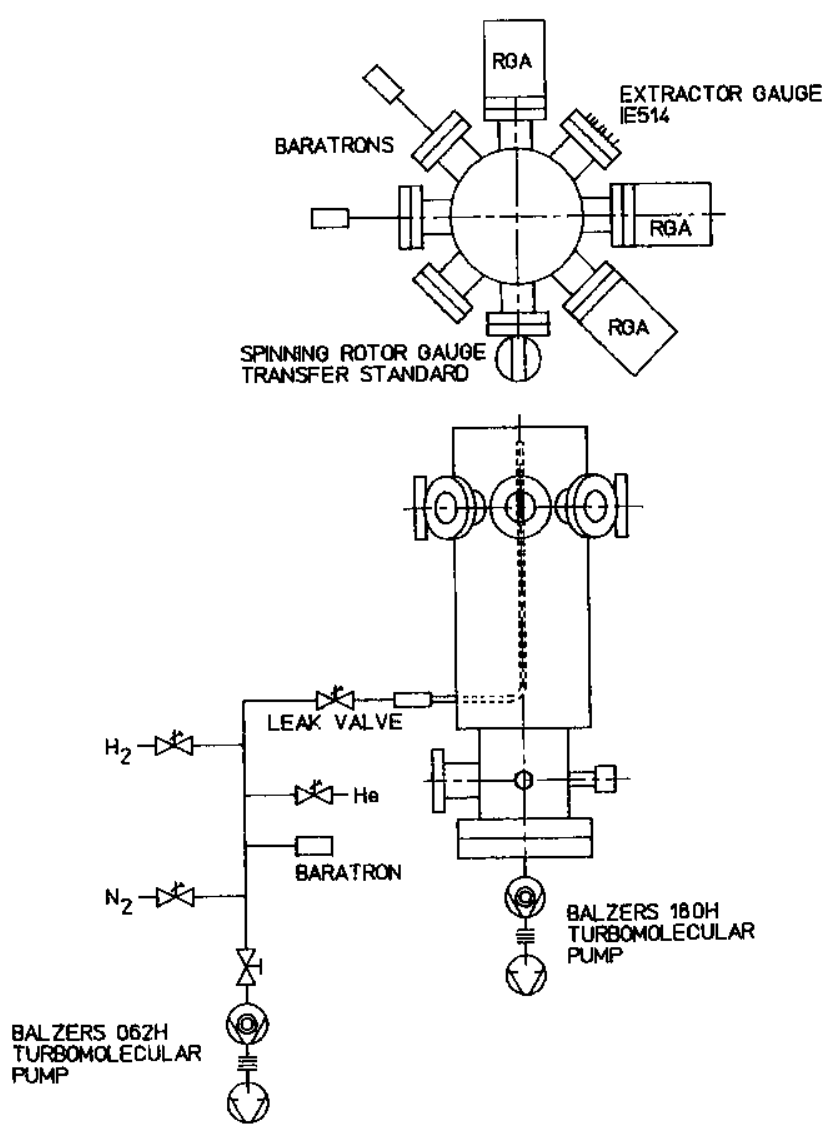

FIG. 1. Schematic of the experinental system. 
TABLE I. Some specifications of tested RGAs.

\begin{tabular}{lccccc}
\hline & $\mathrm{A}$ & $\mathrm{B}$ & $\mathrm{C}$ & $\mathrm{D}$ & $\mathrm{E}$ \\
\hline Filament material & $\mathrm{ThO}_{2} / / r$ & $\mathrm{ThO}_{2} / I r$ & $\mathrm{Y} t / l r$ & $\mathrm{ThO}_{2} / I r$ & $\mathrm{ThO}_{2} / / r$ \\
Electron emission (mA) & 1 & $0-3.5,1^{\mathrm{a}}$ & 1 & $0-2,2^{\mathrm{a}}$ & 2 \\
Electron energy (eV) & 75 & $25-105,70^{\mathrm{a}}$ & 75 & $0-100,100^{\mathrm{a}}$ & 102 \\
lon energy (eV) & 12 & 8 or $12,12^{\mathrm{a}}$ & 12 & $0-15,7^{\mathrm{a}}$ & 10 \\
Focus (V) & $20-200,80^{\mathrm{a}}$ & $0-150,90^{\mathrm{a}}$ & $20-200,80^{\mathrm{a}}$ & $0-150,10^{\mathrm{a}}$ & $0-100,27^{\mathrm{a}}$ \\
Rod length (mm) & 127 & 114.3 & 127 & 100 & 127 \\
Rod diameter (mm) & 6.35 & 6.35 & 6.35 & 6 & 6.35 \\
Field radius (mm) & 2.794 & 2.63 & 2.794 & 2.6 & 2.77 \\
Frequency (MHz) & 3.0 & 2.75 & 1.8 & 2 & 1.78 \\
Multiplier voltage (V) & $0-3000$ & $0-2500$ & $0-3000$ & $0-3000$ & $1000-3000$ \\
Gain & $\sim 10^{4}$ & $\sim 10^{7}$ & $\sim 10^{4}$ & $\sim 10^{6}$ & $\sim 10^{6}$ \\
\hline \hline aDefault values. & & & & &
\end{tabular}

aDefault values.

The main vacuum chamber of the vacuum gauge calibration facility is designed and fabricated by the HPS division of MKS Instruments Inc. as per ISO/DIN 3567 standards. The chamber consists of 2.75 in. CF ports located at the same height and equally spaced around the main cylindrical body. A MKS spinning rotor gauge (SRG) transfer standard, a MKS capacitance diaphragm gauge, and Leybold Inficon Ionivac IE 514. extractor gauges are attached to the vacuum ports. The SRG transfer standard is used to calibrate the extractor gauge in the pressure range $1 \times 10^{-4}-5 \times 10^{-5} \mathrm{~Pa}$. Since the extractor gauges are known to be quite linear down to the $10^{-10} \mathrm{~Pa}$ pressure range, they are used as the transfer
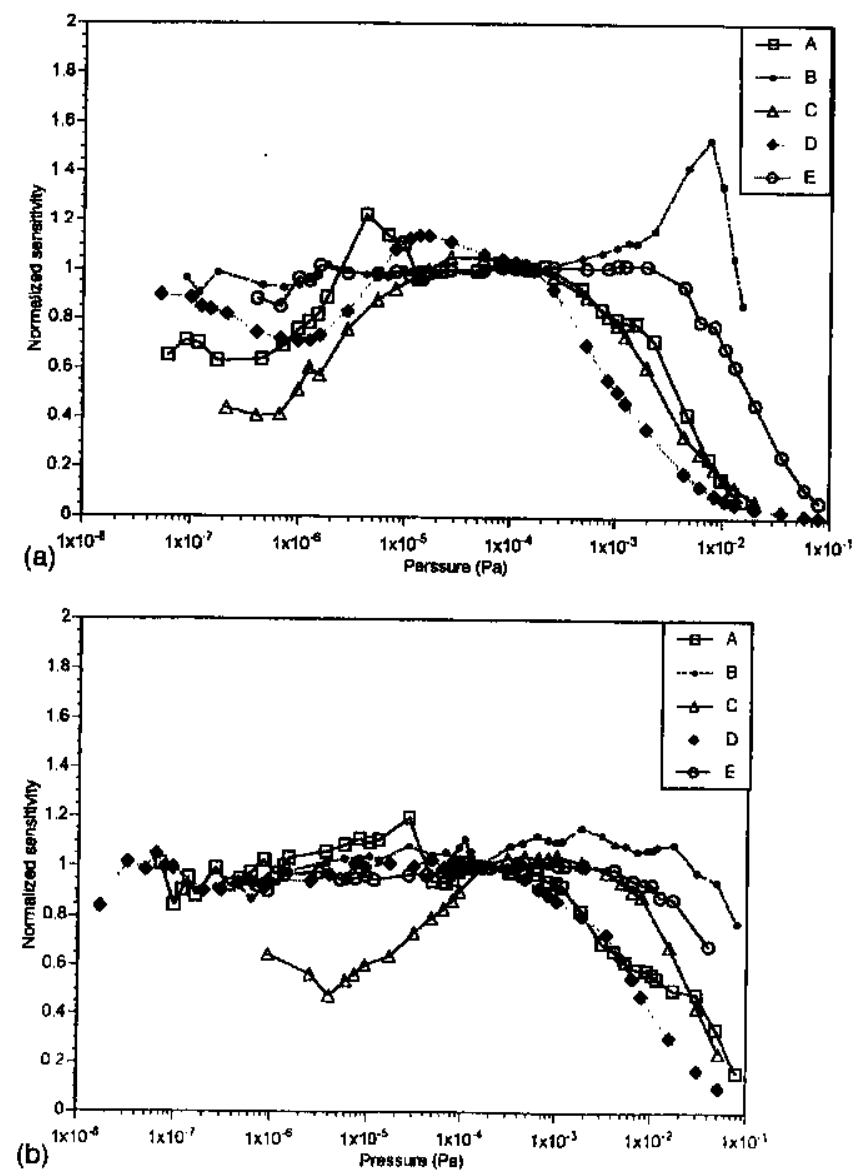

standard. The design of the gas inlet port is such that the calibration gases are introduced at the top of the calibration chamber. The vacuum chamber is pumped by a Balzers $180 \mathrm{H}$ turbomolecular pump and this is backed by a MD4 diaphragm pump. A separate gas inlet system consisting of research grade pure nitrogen, hydrogen, and helium manifolds is connected to the gas inlet port of the calibration chamber through a Varian variable leak. A Balzers TSU $062 \mathrm{H}$ turbomolecular pump is used to evacuate the gas inlet system.

We selected five computer controlled RGAs, A, B, C, D, and $\mathrm{E}$ for these evaluations. The specifications of all these

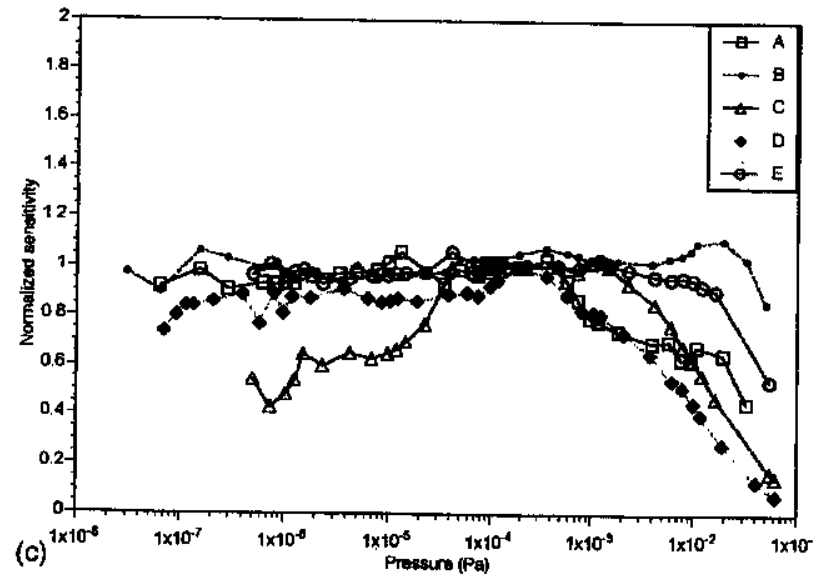

FIG. 2. Normalized sensitivities of RGAs for various gas species; (a) nitrogen; (b) helium; (c) hydrogen. Electron emission/electron energy/ion energy/focus $=1 \mathrm{~mA} / 75 \mathrm{eV} / 12 \mathrm{eV} / 71 \mathrm{~V}, 1 \mathrm{~mA} / 70 \mathrm{eV} / 12 \mathrm{eV} / 90 \mathrm{~V}, 1 \mathrm{~mA} / 75$ $\mathrm{eV} / 12 \mathrm{eV} / 80 \mathrm{~V}, 1 \mathrm{~mA} / 70 \mathrm{eV} / 5.75 \mathrm{eV} / 50 \mathrm{~V}$, and $2 \mathrm{~mA} / 102 \mathrm{eV} / 10 \mathrm{eV} / 27 \mathrm{~V}$ for RGAs A, B, C, D, and E, respectively. 


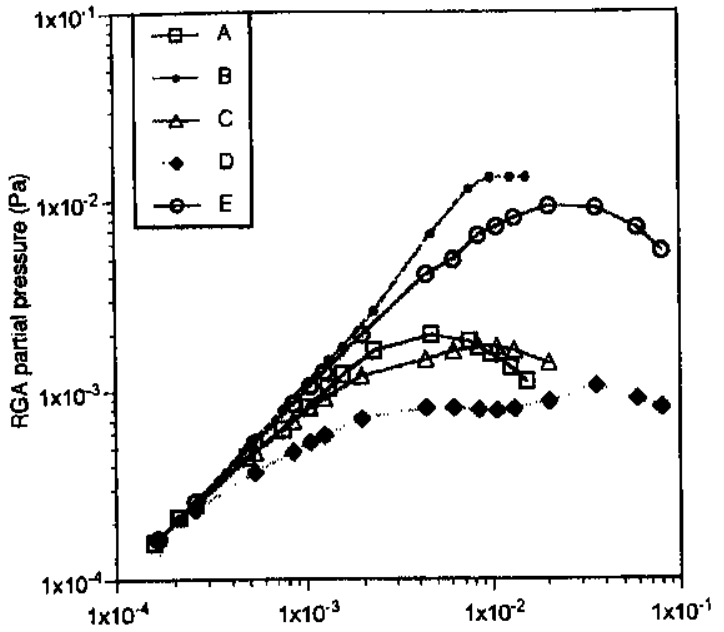

(a)
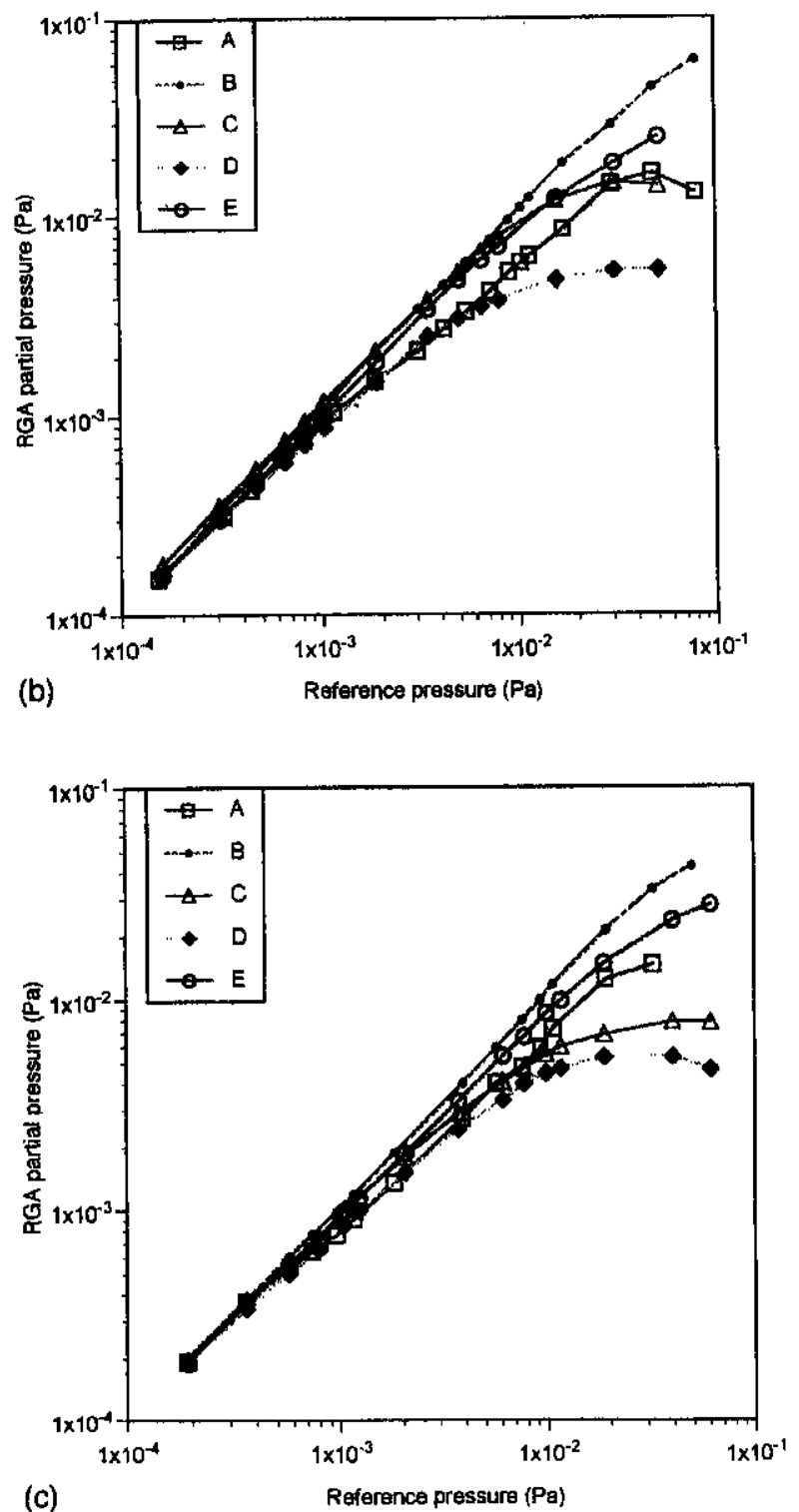

FIG. 3. Linearity of RGAs at high pressure; (a) nitrogen; (b) helium; (c) hydrogen. five RGAs are presented in Table 1 . RGAs $B$ and $E$ are 1-100 AMU units while RGAs C and D are 1-200 AMU units. RGA $A$ is a special unit optimized for low mass range from 1 to $50 \mathrm{AMU}$. The emission current, electron energy, and ion energy settings are fixed for analyzers $A, C$, and $E$ by the vendor. Whereas the user has the option of varying the focus voltage of these three RGAs. The user has the access for changing all the ion source electrical parameters for RGAs B and D.

A direct pressure comparison method is used for these investigations as per the AVS recommended practice. ${ }^{10} \mathrm{SRG}$ is the reference gauge for pressures above $1.3 \times 10^{-4} \mathrm{~Pa}$ and the extractor gauge is the reference for pressures below $1.3 \times 10^{-4} \mathrm{~Pa}$. Sensitivity of the RGAs is calculated as

$$
\mathrm{S}_{i}=\left(I_{i}-I_{0}\right) /\left(P_{i}-P_{0}\right),
$$

where $I i$ is the RGA reading at a reference pressure $P_{i}$, and $I_{0}$ is the background signal corresponding to a pressure of $P_{0}$.

Before each test, the calibration chamber is pumped down to about $4 \times 10^{-8} \mathrm{~Pa}$. The RGAs are calibrated at a pressure of $\sim 1.6 \times 10^{-4} \mathrm{~Pa}$ for the Faraday cup and at a pressure of $\sim 1.6 \times 10^{-6} \mathrm{~Pa}$ for the electron multiplier detector. The resolution is adjusted to a peak width of 1 AMU at $10 \%$ peak height for the hydrogen main peak $M / Q=2$ and for the nitrogen main peak $M / Q=28$.

\section{RESULTS AND DISCUSSIONS}

\section{A. Sensitivity and linearity}

Most of the reported sensitivity data in the literature has been for argon since it is a stable gas species. We have selected nitrogen, helium, and hydrogen as the test gases for our investigations. Nitrogen is chosen because it is the gas that vendors mostly use for calibrating the RGAs. Helium is selected since it is universally used for leak detection purposes. And, finally, hydrogen is chosen as it is the most common and the largest peak in the UHV systems. For comparing the sensitivity of RGAs with various gas species, the ion source electrical parameters, viz. electron emission current, electron energy, ion energy, and focus voltage, are either selected to be the default values as recommended by the vendor or optimized for each instrument. The chosen values for these parameters as well as the normalized sensitivity data (normalized at the calibration pressure of $1.6 \times 10^{-4} \mathrm{~Pa}$ ) for nitrogen, helium, and hydrogen are presented in Fig. 2.

The RGAs may be grouped into two categories based on the normalized sensitivity data for nitrogen. RGAs A, C, and $\mathrm{D}$ may be named as group $\mathrm{X}$ with $\mathrm{B}$ and $\mathrm{E}$ as group $\mathrm{Y}$. The nitrogen sensitivity of the group X RGAs is poor compared to that of group $Y$. The optimization efforts of the ion source parameters of RGA D, namely, the electron emission current, electron energy, and ion energy, did not produce any appreciable improvement in the sensitivity for nitrogen. The reasons for this nonlinearity are not very clear and are likely to depend on the ionization probability of the particular ion source. Further, the high fragmentation factor at low pressures may be a contributing factor and will be discussed 
TABLE II. Relative sensitivities of hydrogen and helium to nitrogen.

\begin{tabular}{llllll}
\hline & $\mathrm{A}$ & $\mathrm{B}$ & $\mathrm{C}$ & $\mathrm{D}$ & $\mathrm{E}$ \\
\hline Helium & 0.3 & 0.33 & 0.3 & 0.5 & 0.3 \\
Hydrogen & 0.7 & 1.5 & 0.8 & 1.6 & 0.7 \\
\hline
\end{tabular}

later. The nitrogen sensitivity of RGA B starts increasing at a pressure of about $10^{-3} \mathrm{~Pa}$. This deviation from the linearity is due to the positive ion space charge repulsion in the ionization volume. ${ }^{11}$ Most of the RGA manufacturers calibrate their RGAs with nitrogen at a pressure of $10^{-4} \mathrm{~Pa}$. Since the nitrogen sensitivities of group X RGAs begin to deviate below a pressure of $10^{-5} \mathrm{~Pa}$, the measurement accuracy of these instruments will suffer below $10^{-5} \mathrm{~Pa}$. As a consequence, the relative sensitivities of these instruments for other gases at low pressures also will be inaccurate. The group Y RGAs show very good sensitivity for both helium and hydrogen to very high pressures of $10^{-2} \mathrm{~Pa}$. The sensitivity of RGAs A and D for helium and hydrogen is fairly good in comparison to that of RGA $\mathrm{C}$.

The high-pressure linearity data for all five RGAs is presented in Fig. 3 for nitrogen, helium, and hydrogen. All the RGAs show excellent linearity up to a pressure of $10^{-2} \mathrm{~Pa}$ for both helium and hydrogen. This excellent linearity for helium and hydrogen may be due to their small ionization cross sections and, hence, the reduced space charge effects at high pressure in comparison to nitrogen. Further, the throughput of the quadrupole filter is higher for hydrogen and helium in comparison to nitrogen, thereby making up for the low ionization efficiency of the light gases. However, the linearity of the group $Y$ RGAs starts deteriorating above $10^{-3} \mathrm{~Pa}$ for nitrogen.

\section{B. Relative sensitivity and the function of focus voltage}

As mentioned earlier, most of the RGAs are calibrated with nitrogen gas and the sensitivity data is quoted with reference to nitrogen. The sensitivity of these instruments for other gases is different, as in the case of ion gauges, and is generally measured with reference to nitrogen. The ratio of the absolute sensittivity for a different gas to its absolute sensitivity to the reference gas (nitrogen) is defined as relative sensitivity. The relative sensitivities of all the RGAs for hydrogen and helium are presented in Table II with the same operating parameters as used for obtaining the sensitivity data shown in Fig. 2 for nitrogen. Except for RGA D, the instruments have a relative sensitivity factor of $\sim 0.3$ for helium. The relative sensitivity factor for hydrogen is quite different from one instrument to the other and is higher than for helium.

The focus voltage (also known as the extraction voltage) may also influence the ionization probability, ion detection efficiency, and relative sensitivity. The focus voltage is the only parameter that the user can change in all five instruments. The effect of focus voltage on the ion current for various instruments with different gases and the relative sensitivity factors has not been reported in the literature. We have investigated the effect of focus voltage on the ion current, gas species, and relative sensitivity factors at a pressure (nitrogen) of $10^{-4} \mathrm{~Pa}$ and the results are presented here. Figure 4 gives the ion current as a function of the focus voltage for RGAs C, D, and E. The ion current of RGA $E$ is insensitive to the focus voltage at a value higher than $15 \mathrm{~V}$. The ion current of instrument $C$ is very sensitive to the focus voltage in the range $65-75 \mathrm{~V}$. The ion current gradually increases with decreasing focus voltage below $65 \mathrm{~V}$ and the current declines as the focus voltage is increased above $75 \mathrm{~V}$.

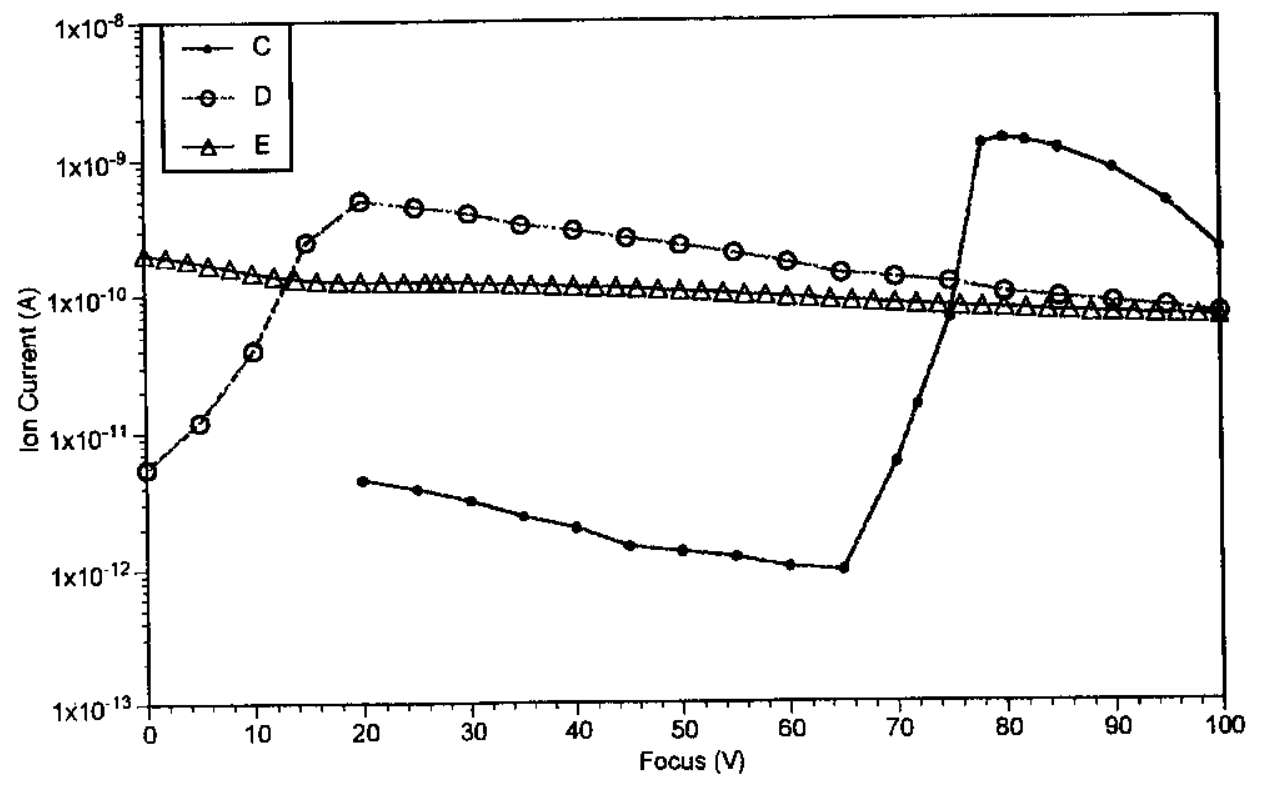

FuG. 4. Relationship between focus voltage and ion current for different RGAs for nitrogen at $1.5 \times 10^{-4} \mathrm{~Pa}$. 


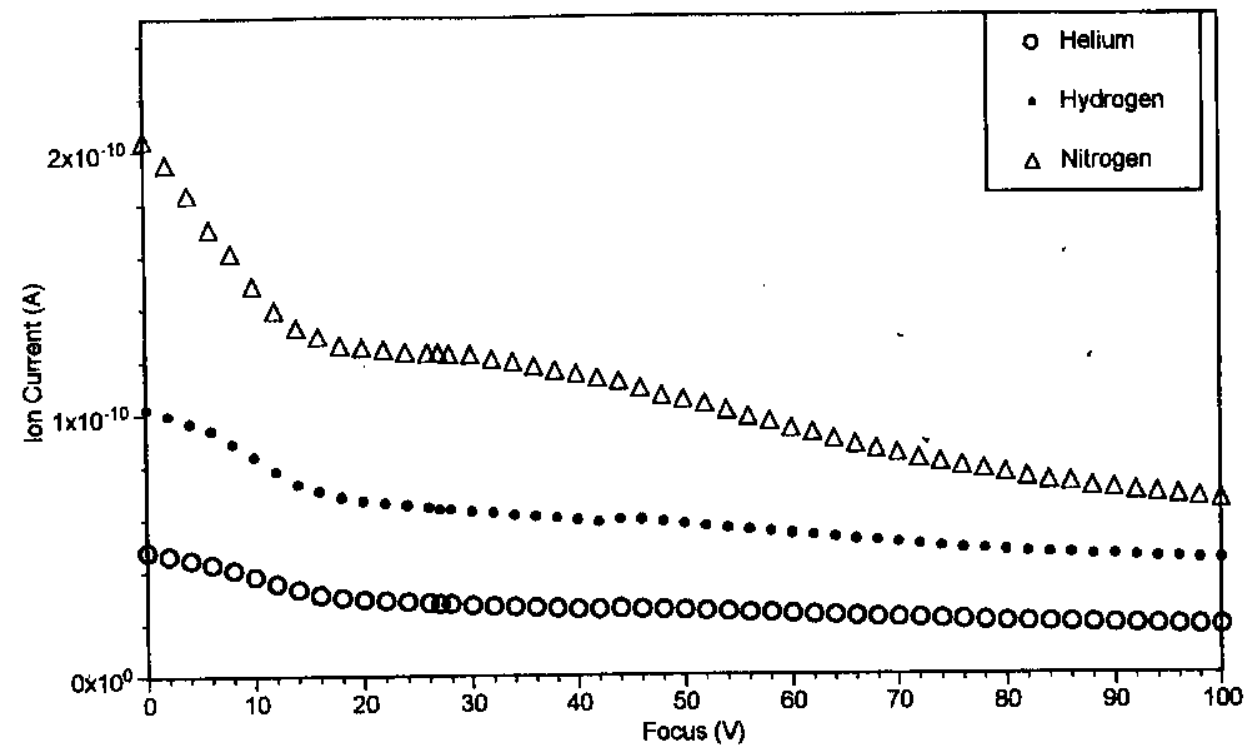

FIG. 5. Relation between focus voltage and lon current for different gas species with RGA E.

The ion current of instrument $\mathrm{D}$ decreases with increasing focus voltage value above $20 \mathrm{~V}$. In general, this decreasing trend in ion current with increasing focus voltage can be attributed to the repulsion of electrons from the ionization space, thereby, reducing the ionization probability.

The effect of focus voltage on the ion currents for nitrogen, helium, and hydrogen is shown in Fig. 5 for RGA E. The ion current for the three gas species declines at different rates as the focus voltage is increased. This decrease is directly proportional to the mass of the gas species and shows a mass discrimination towards higher mass ions. The relative sensitivity factors of hydrogen and helium for RGA $E$ are presented in Fig. 6 as a function of focus voltage.

\section{Investigation of fragmentation factor}

The fragmentation factor or cracking pattern of the gases depends on the instrument structure and its operating parameters. ${ }^{12}$ The published data show large variation in the fragmentation factors. As an example, the abundance of the fragmentation peak of $M / Q=14$ for nitrogen can vary anywhere from $5 \%$ to $14 \%$ for different instruments. We have

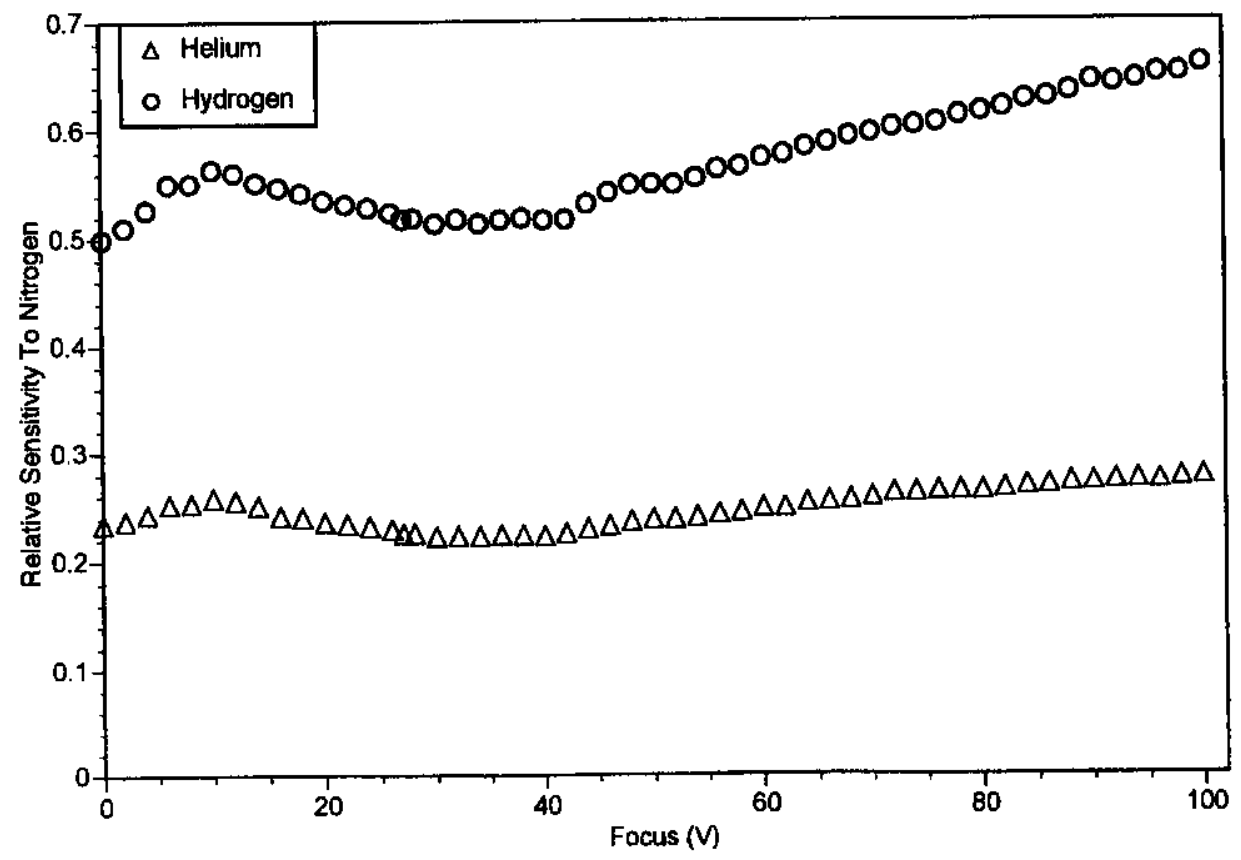

FrG. 6. Relation between relative sensitivity and focus for $\mathrm{He}, \mathrm{H}_{2}$ with $\mathrm{RGA}$ E. 


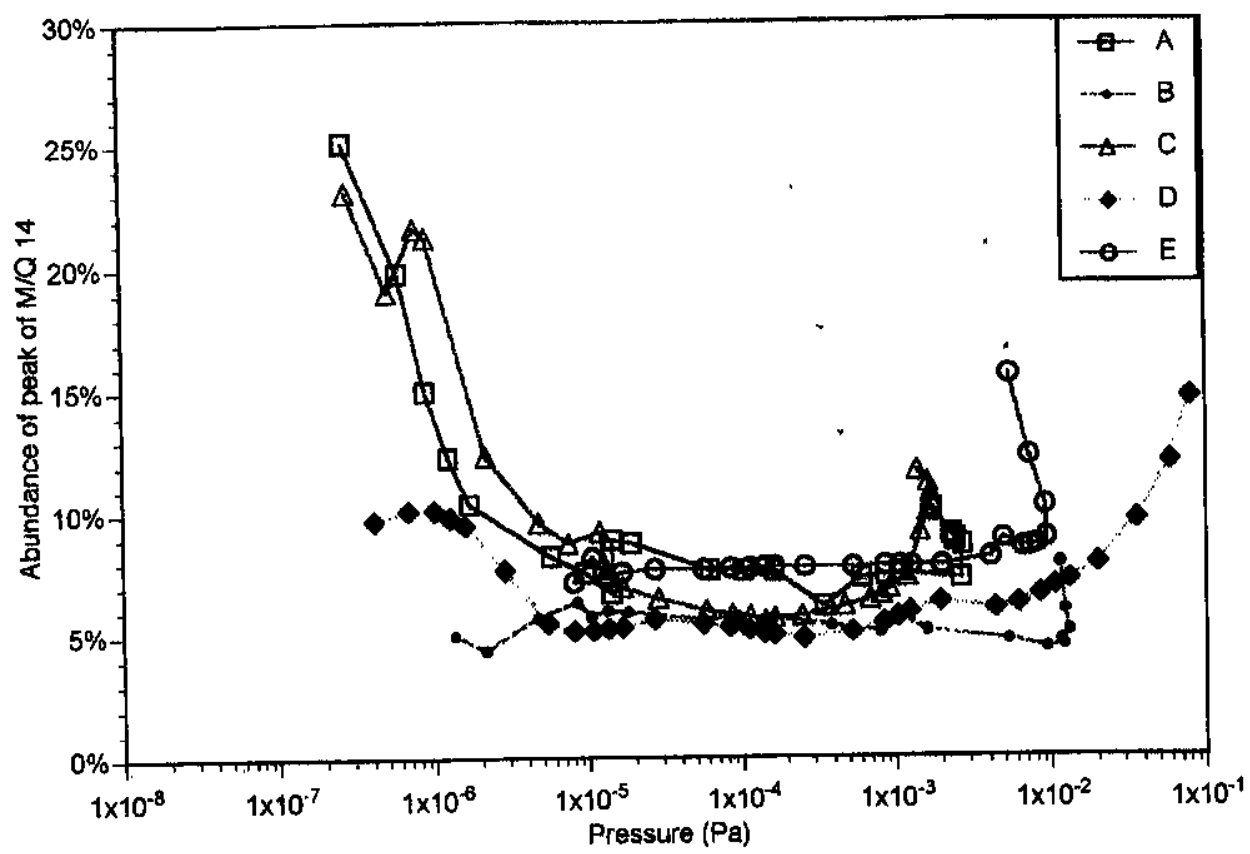

Fig. 7. Abundance of fragmentary peak of $M / Q=14$ for ntirogen.

investigated the cracking pattern for nitrogen and hydrogen. It is known that the electron emission current, electron energy, ion energy, and the focus voltage influence the fragmentation factor. However, electron emission current plays a major role in the fragmentation factor. The abundance of the peak at $M / Q=14$ is shown in Fig. 7 for all the instruments with the same operating parameters as given in Fig. 2. The value of the abundance of the fragmentation peak at $M / Q$ $=14$ for nitrogen mostly varies from $5 \%$ to $8 \%$ in the pressure range $10^{-5}-10^{-2} \mathrm{~Pa}$ for all the instruments. At pressures above $10^{-3} \mathrm{~Pa}$, the fragmentation factor at $M / Q=14$ increases for RGAs $A$ and $C$, while this increase takes place above $10^{-2} \mathrm{~Pa}$ for the rest of the instruments. The abundance factor increases from $6 \%$ to $25 \%$ in the cases of RGAs A and $\mathrm{C}$, and the increase for RGA D is from $5 \%$ to $10 \%$ at pressures below $10^{-5} \mathrm{~Pa}$.

The fragmentation factor at $M / Q=14$ increases from $6 \%$ to $10 \%$ as we increase the electron emission current from 1 to $2 \mathrm{~mA}$ for RGA D, and is shown in Fig. 8. A similar increase in emission current from 1 to $2 \mathrm{~mA}$ changes the fragmentation factor at $M / Q=14$ from $6 \%$ to $14.5 \%$ in the case of instrument B. An ion energy increase from 8 to 12

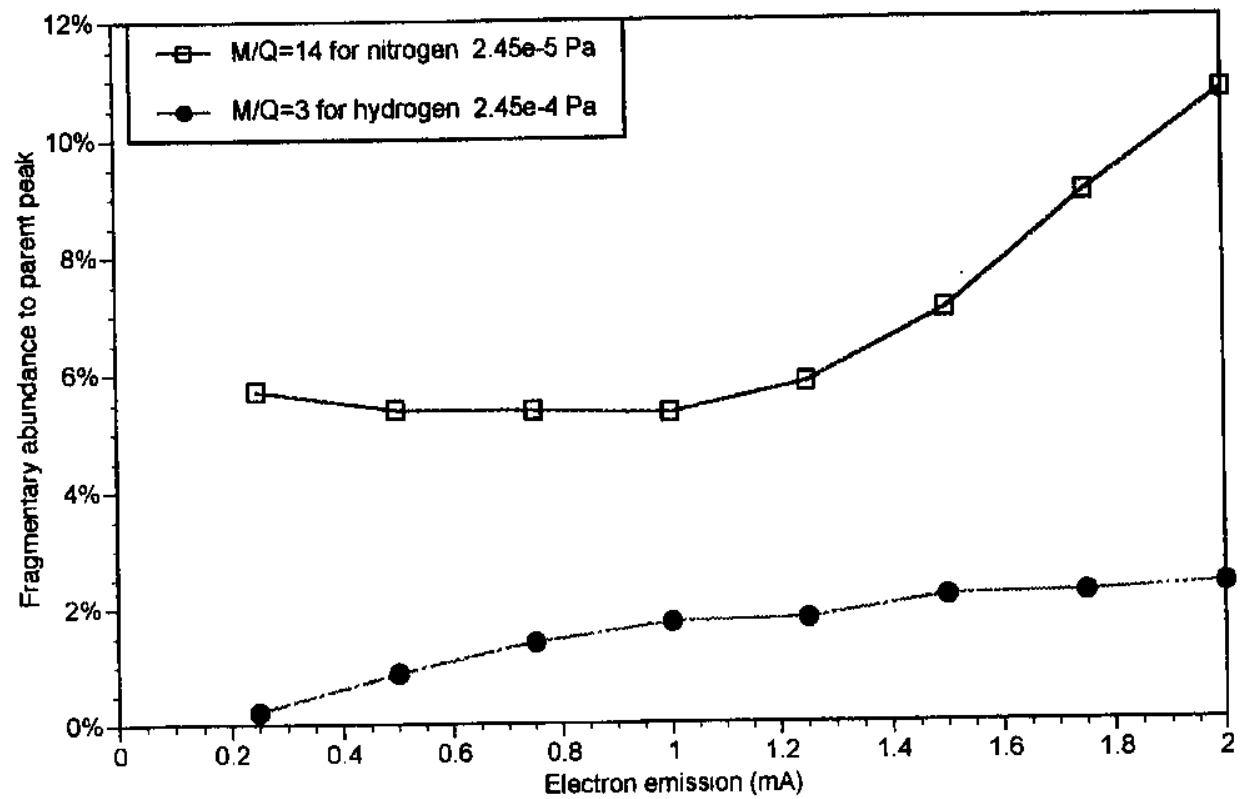

FIG. 8. Relation between fragmentary peak abundance with electron emission current for RGA D. 
$\mathrm{eV}$ increases the abundance at $M / Q=14$ by less than $1 \%$ again for instrument $B$. The fragmentary abundance at $M / Q=3$ increases to $2.5 \%$ as we increase the electron emission current from 0.2 to $2 \mathrm{~mA}$, and is shown in Fig. 8 for RGA D. We believe that the high cracking ability of RGAs $\mathrm{A}, \mathrm{C}$, and $\mathrm{D}$ for atomic species weakens the stable production of the parent peak for nitrogen and many other molecular species at low pressures.

\section{SUMMARY}

The evaluation of the computer controlled RGAs has been carried out over a period of five months. Obviously, it is impossible to provide comprehensive test data on the investigated RGAs in such a short time. We recognize that some of the instruments may provide a linear response after careful selection of the best combinations of the operating parameters. We also believe that the default parameters may not be suitable for all applications.

The linearity behavior of RGAs for different gas species is not uniform. At high pressures the linearity of RGAs is better for low mass gas species ( $\mathrm{He}$ and $\mathrm{H}_{2}$ ) by an order of magnitude in pressure compared to higher AMU gas species. The sensitivity of RGAs A, C, and D deviates from linear behavior at low pressures for nitrogen in comparison to RGAs B and E. The sensitivity of RGA C is poor in comparison to every other instrument and for all the test gases at low pressures. The relative sensitivity of RGA D for helium is the highest. The relative sensitivity of RGAs for hydrogen varies from 0.7 to 1.6 . The electron emission current seems to play a major role in the fragmentation factor of the RGAs.
The fragmentation factors seem to increase both at high and low pressures. With appropriate calibrations, the computer controlled low cost RGAs can be used for quantitative analysis of UHV systems. Further, they are extremely useful for leak detection of vacuum systems.

\section{ACKNOWLEDGMENTS}

This work wass supported by the U.S. Department of Energy under Contract No. DE-AC05-84ER40150. One of the authors, C. Dong, is a visiting Scholar from Lanzhou Institute of Physics, P. R. C. and acknowledges the support of his government for the Scholarship.

'M. G. Rao, J. Vac. Sci. Technol. A 11, 1598 (1993).

${ }^{2}$ W. R. Balnchard, P. J. McCarthy, H. F. Dylla, P. H. LaMarche, and J. E. Simpkins, J. Vac. Sci. Technol. A 4, 1715 (1986).

${ }^{3}$ P. H. Dawson, Quadrupole Mass Spectrometry and Its Applications (AIP Press, New York, 1995).

${ }^{4}$ J. H. Batley, Vacuum 37, 659 (1987).

${ }^{\mathrm{J}} \mathrm{J}$. H. Leck, Total and Partial Pressure Measurement in Vacuum Systems (Blackie, Glasgow, 1987).

${ }^{6}$ P. H. Dawson, J. Vac. Sci. Technol. A 4, 1709 (1986).

${ }^{7}$ L. Lieszkovszky, A. R. Filippelli, and C. R. Tilford, J. Vac. Sci. Technol. A 8; 3838 (1990).

${ }^{8}$ W. E. Austin, J. H. Leck, and J. H. Batey, J. Vac. Sci. Technol. A 10, 3563 (1992).

${ }^{9}$ W. E. Austin, Fu Ming Mo, Jin Man Yang, and J. H. Leck, J. Vac. Sci. Technol. A 5, 2631 (1987).

${ }^{10} \mathrm{~J}$. A. Basford, M. D. Boeckmann, R. E. Elleson, A. R. Fillippelli, D. H. Holkeboer, L. Leiszkovszky, and C. M. Stupak, J. Vac. Sci. Technol. A 11. A22 (1993).

${ }^{11}$ M. C. Cowen, W. Allison, and J. H. Batey, J. Vac. Sci. Technol. A 12, 228 (1994)

${ }^{12}$ F. M. Mao and J. H. Leck, Vacuum 37, 675 (1987). 